\title{
Assistive Technology: Does it Really Help Blind People?
}

\section{Tianyu Chen}

University of Army police sergeant

Abstract: This report discusses how assistive technology affect the blind people's life. Bind assistive technology would enhance blind people's independent living ability and give blind people multiply ways to play and identify products. Besides, this report also examines the negative aspect that assistive technology would bring to the blind people Keywords: Assistive Technology; Bind People; Living Ability; Safety Issues

\section{Introduction}

Over the past decade, evolving technologies such as information technology have change the daily lives for people. However, there is a kind of people who are visually impaired and it is hard for them to use the electronic technology. Take the United States as an example, there are approximately one million blind people in the US (Robert, 2003). Due to this situation, blind people need to have some technologies that can help them access the digital world.

Blind Assistive technology is a collection of devices or systems which allow disabilities to do the task that they would unable to do before or increase the safety and convenient when blind people doing the task (Cowan \& TurnerSmith, 1999). It includes many different types, such as mobility aids, hearing aids, furniture or daily living aids and so on (Creadie \& Tinke, 2005) . There have many different products now in the market and many blind people are trying to use them. However, due to the user experience and some researchers argument, people really curious about whether assistive technology is helpful for blind people or not. This essay will argue that the positive aspects of assistive technology are overweight its negative effects.

\section{Assistive technology enhance blind people's independent living ability}

Firstly, assistive technology using audible signals to warn the disabilities. Some new products such as 'Boil Alert' and EZ Fill liquid level indicator would sound an alarm when the water in the pot is boiling or the glass reaches a specified level (Robert, 2003). Blind people are sensitive to the sound, this technology provides them they way to do some simple things such as turning on the water to have a bath even there have no people to help them. Moreover, the audible signal technology can also use the furniture and stairs. Therefore, when blind people are closed to the sofa or stairs, the warning bell would alarm them there has a barrier and keep the blind people in a safety place.

Secondly, some products such as 'blind spot' and blind navigation system allow people to intelligent find obstacles and the way to the destination. Blind spot is a kind of intelligent blind cane which can detect and alarm the users when they meet some objects in the street. It has the artificial intelligence voice system which would tell the users when they need to cross the road or close to the friend they want to meet. This allows them do not need to walk in the blind road and also not miss their friend. In addition, the blind navigation system provides visually impaired individuals a portable and self-contained system which allows them to travel through unfamiliar and familiar place without other people's guides (Jack, Reginald \& Roberta, 1998). Thus, within these products, blind people can live almost as a normal person and less dependent on others to live.

\section{Assistive technology giving blind people multiply ways to play and identify}

Copyright (C) 2020 Tianyu Chen

doi: 10.18282/le.v9i7.1508

This is an open-access article distributed under the terms of the Creative Commons Attribution Non-Commercial License

(http://creativecommons.org/licenses/by-nc/4.0/), which permits unrestricted non-commercial use, distribution, and reproduction in any medium, provided the original work is properly cited. 


\section{products}

The most important aspect that assistive technology give is the accessibility for blind people to use the computer and other mobile devices. The external haptic keyboard is a special keyboard which has the points that blind people can touch and identify the meaning of the related letter. When they push the button, it would also speak the letter and allows them to check the spell (Shinohara \& Tenenberg, 2009). Another touch screen technology named Slide Rule gives blind people the method to use the touch phone. This product lets users only using the different gestures to correspond to the different phone functions (Shaun, Jeffrey \& Jacob, 2008). Therefore, blind people can also use the electronic devices to surf the internet and talk to other people online.

Moreover, some mobile applications such as 'be my eyes' can link the volunteers around the world to help the blind people identify the products. When people using this application, the volunteers can see the things which blind people hold and tell them what is it and even how to use it. So, it will help blind people choose the goods, enhance the social participation and also provide the channel for normal people to understand them.

\section{Negative aspects of assistive technology}

It is true that assistive technology sometimes may have negative effects. Many assistive technology products are different from the normal electronic devices, when blind people using them, these products would make them very 'eyecatching'. According to Shinohara \& Jacob (2011), they point out that if the products of assistive technology are more visible and have strange looking, they look more attracting than others. However, most blind people do not want others to pay attention to them. Thus, this makes them feel they are different from the normal people and may cause inferior mentality. Moreover, some assistive technology products lack stability. Take the intelligent blind cane as an example, sometimes the light and other factors may lead the barriers in front of the users not been identified (Creadie \& Tinker, 2005). Therefore, blind people may strike by these obstructions which may cause the safety issues. This is also the reason why some blind people unwilling to using assistive technology products.

\section{Future perspective of assistive technology}

According to the research, more and more assistive technology are produced in this decade. With the use of most products, blind people's life become easier and more comfortable. However, there still have space for the innovators to develop. Firstly, they should improve the products' quality and safety, make sure the product can achieve its functions and have fewer safety issues. Secondly, the future products should have better interaction with the users, such as optimizing the product exterior design and make it as similar as the product for the ordinary people. This would make the technology more easy to accept by the blind people and can really solve their problems.

In conclusion, blind assistive technology has many benefits that can help blind people in their daily life experience and also enrich their entertainment and study. It has some disadvantages but would be solved in the future. Therefore, governance and researchers can do more work on this technology and make it more powerful to help the disabilities.

\section{References}

1. Cowan, D,. \&Turner-Smith, A. (1999). The role of assistive technology in alternative models of care for older people. Royal Commission on Long Term Care. Vol. 2, 325-46.

2. Creadie, C., \& Tinker, A. (2005). Ageing and Society. United Kingdom: Cambridge University Press.

3. Jack, ML., Reginald, GG., \& Roberta, LK. (1998). Navigation system for the blind: auditory display modes and guidance. Massachusetts Institute of Technology. April, vol. 7(2), 193-203.

4. Robert, WM. (2003). Auditory assistive devices for the blind. Proceedings of the 2003 International Conference on Auditory Display. July 6-9, 271-275.

5. Sara, HB., Kanevsky, D., Edward, EK., \& West, F. (2011). Method and system for blind spot identification and warning utilizing portable and wearable devices. International Business Machines Corporation. Mar, 1-9.

6. Shaun, kk., Jeffrey, PB., \& Jacob, OW. (2008). Slide rule: making mobile touch screens accessible to blind people using multi-touch interaction techniques. ASSETS. October, 13-15.

7. Shinohara, K., \& Tenenberg, J. (2009). A blind person's interactions with technology. Communications of the ACM. August, vol. 52, 58-66.

8. Shinohara, K., \& Jacob, OW. (2011). In the shadow of misperception: assistive technology use and social interactions. HCI for All. May, 705-714. 